\title{
Producción de sujetos y espacios para el ejercicio de violencia feminicida en Puebla, México ${ }^{1}$ \\ Production of Subjects and Spaces for the Practice of FeminicidalViolence in Puebla, Mexico
}

\author{
Luis Fernando Gutiérrez Domínguez \\ Benemérita Universidad Autónoma de Puebla, Puebla de Zaragoza, México \\ luis.gutierrezd@correo.buap.mx|https://orcid.org/0000-0002-1087-9536 \\ Nayeli Sánchez Mora \\ Benemérita Universidad Autónoma de Puebla, Puebla de Zaragoza, México \\ nayeli_snm@hotmail.com|https://orcid.org/0000-0001-6620-0046
}

Recibido: 31 de agosto de 2020. Aprobado: 22 de septiembre de 2020.

DOI: $10.25100 /$ lamanzanadeladiscordia.v15i2.10554

Artículo de investigación

\begin{abstract}
¿Cómo citar este artículo? /Howtoquotethisarticle?
Gutiérrez Domínguez, Luis Fernando, y Sánchez Mora, Nayeli. (2020). Producción de sujetos y espacios para el ejercicio de violencia feminicida en Puebla, México. La Manzana de la

Discordia, 15(2),1-29. 10.25100/lamanzanadeladiscordia.v15i2.10554
\end{abstract}

\footnotetext{
${ }^{1}$ Esta investigación se inscribe en el marco del Programa de Apoyo a la incorporación de nuevos profesores de tiempo completo, convenio 511-6/17-13245, BUAP-PTC-523, así como del programa de Servicio social de la Facultad de Ciencias de la Comunicación (Folio 105941, Período 1-2019), instancias a las que agradecemos su respaldo para la presentación de este artículo.
} 


\title{
Resumen
}

El modelo económico hegemónico, centrado en la creciente incorporación de mujeres al espacio público-productivo y la paulatina retirada de los hombres del mismo, se constituye en punto de partida conceptual desde el cual se observan formas concretas de desigualdad en las relaciones sociales de género, en este caso, prácticas de violencia exacerbada como el feminicidio. Mediante la revisión de noticias en diarios locales del estado de Puebla, México, durante los años 2018y 2019, de manera reflexiva planteamos que sectores económicos de población con inserción precaria al mercado laboral, dan cuenta de la ubicación del fenómeno feminicida en ese espacio sociopolítico; asimismo,enfatizamos que en los medios de comunicación digital se manifiestan representaciones estereotipadas de género que sitúan el escenario feminicida como espectáculo morboso que, al paso del tiempo deriva en la pérdida de su atractivo y de la atención pública, sin que por ello disminuyan los casos letales de feminicidio.

Palabras clave: Espacio generizado; Geografía feminista; Producción mediática; Violencia feminicida.

\begin{abstract}
The hegemoniceconomic model, focused on the increased female incorporation to the publicproductive space, as well as on the gradual male retreatfromit, constitute the conceptual starting point for observing concrete shapes of inequality in gender relationships, such as those exacerbated violent practices against women in the form of feminicide. By reviewing news in local digital newspapers in the state of Puebla, Mexico,during2018and 2019, we declare in a reflexive way that productive economic sectors defined by their precarious insertion labor conditions show that feminicide pertains to that kind of sociopolitical space; We also emphasize that in digital mass media are deployed stereotyped gendered representations setting the feminicide scenario as a morbid spectacle that, with the pass of time drives to the diminution of its attractiveness and lack of public attention, without reducing lethal feminicide numbers.
\end{abstract}

Key Words: Gendered Space; Feminist Geography; Mass Media Production; Femicide Violence. 


\section{Introducción}

En el contexto de la investigación en curso, Fragmentación territorial y violencia de género en Puebla $^{2}$, cuyo objetivo central es el conocimiento de las adversidades económicas y políticas que las mujeres afrontan desde su condición de género ${ }^{3}$ en el marco de la globalización neoliberal, planteamos que el espacio es resultado de un proceso de producción histórico, político, económico, geográfico y simbólico, intervenido por acciones estatales, patriarcales, androcéntricas y misóginas, desde el cual se pueden visualizar tensiones, contradicciones y ambigüedades en las relaciones sociales de género entre hombres y mujeres. El feminicidio, fenómeno referido en esta presentación, ejemplifica ello.

Como podrá observarse, las formas de representación del mundo se encuentran profundamente conectadas con las condiciones materiales de la existencia social, de modo tal que estas se solapan mutuamente y producen prácticas de alta complejidad que exigen observación sistemática para su comprensión en un primer momento, y para el cambio social como horizonte de futuro (Fraser, 2020; Falquet, 2014; Pérez, 2014; Picchio, 2005; Del Valle, 1997; Eisenstein, 1997; Rose, 1993).

A tono con la promesa liberadora del feminismo de la segunda ola, pensamos en tres dimensiones de injusticia de género a ser superadas: económica, cultural y política (Fraser, 2008); ellas sintetizan la vida contemporánea, la cual se traduce en pobreza de tiempo, en desequilibrio familia-trabajo, en agotamiento social, y en la llamada crisis de cuidados, que produce afectaciones directas a mujeres como sujetos tradicionalmente asignados a esas tareas, al tiempo que dificulta el proceso de reproducción social, considerado por Nancy Fraser (2020) como la contradicción social del capitalismo, modo hegemónico de gestión de la vida que se opone, per se, a la solución de tales adversidades.

\footnotetext{
${ }^{2}$ Esta investigación se inscribe en el marco del Programa de Apoyo a la incorporación de nuevos profesores de tiempo completo, convenio 511-6/17-13245, BUAP-PTC-523, así como del programa de Servicio social de la Facultad de Ciencias de la Comunicación (Folio 105941, Período 1-2019), instancias a las que agradecemos su respaldo para la presentación de este artículo.

${ }^{3}$ Empleamos condición de género (Lagarde, 2005), como categoría analítica capaz de relevar la estructura subyacente de exclusión y asimetría que determina a las mujeres en sus relaciones con los hombres en tanto sujetos genéricos, para mostrar sus efectos simbólicos y prácticos dentro de un sistema social, histórico, económico y político, configurado androcéntricamente, que admite la dicotomía biología/sociedad como incontrovertible.
} 
Antes de abordar la noción de feminicidio, queremos hacer explícito el sentido que empleamos de manera constante a lo largo de la exposición para referirnos al poder, el cual, identificamos como una forma de relación social configurada a lo largo del tiempo y del espacio que, tematizada en clave de género, ha supuesto el ordenamiento de la vida social sobre la base de la capacidad del genérico masculino para subordinar, excluir, discriminar, oprimir y explotar al genérico femenino. Dado su carácter histórico, también asumimos que es susceptible de transformación a partir de desmontar los supuestos ideológicos que lo soportan ${ }^{4}$.

\section{¿Femicidio o feminicidio? Matices conceptuales a considerar}

La jerga criminológica que ha cubierto el fenómeno de la violencia contra las mujeres, proviene de históricas subjetivaciones patriarcales, al grado que, de acuerdo con necesidades del orden social androcéntrico, minimiza aquellas acciones que suprimen las vidas de las mujeres (DeKeseredy, 2011; Russell, 2006b). Al final, en lo que se sitúa como un mecanismo que perpetúa la violencia como reflejo normal de lo humano, queda la sensación de uso común de que, si se trata de muertes violentas, más hombres que mujeres mueren cada año, de ahí que no debería sobresaltarnos el hecho de las muertes femeninas, sino buscar medidas que eviten las de ellos para, eventualmente, reducir las de ellas. Jill Radford (1992) se preguntaba por la importancia de comprender por qué el asesinato de una mujer singular podía despertar interés social (o morbo), mientras que el asesinato colectivo de mujeres, caracterizado como misógino, quedaba velado a la preocupación social, política y académica.

La insuficiencia explicativa de tales muertes violentasimpulsó a Diana Russell a trazar su historicidad, de la cual destacamos lo siguiente: 1801 fue la primera vez que se empleó el término feminicidio en una obra literaria inglesa; para 1848 formó parte del lenguaje judicial, indicando que se consideraba un crimen. Se trataba de una definición general que requería precisiones; de esta manera, inicialmente lo definió como "el asesinato de mujeres por hombres por ser mujeres" (Russell, 2006a, p. 76). Con el apoyo de evidencia etnográfica proveniente de diferentes regiones del mundo, pasó a una definición más articulada y compleja, en la cual está presente la mediación

\footnotetext{
${ }^{4}$ A este respecto, puede seguirse el recuento que María de la Fuente Vázquez (2015) elabora sobre la discusión en torno al poder y su centralidad al interior de los feminismos a partir de la década de 1980, así como sus límites y alcances en términos de la configuración de las mujeres como sujeto y la correspondiente exigibilidad del reconocimiento político y la redistribución de la riqueza.
} 
misógina y sexista, y resulta irrelevante la intencionalidad o ausencia de la misma en la supresión de la vida de las mujeres, aunque deba valorarse el tipo de vínculo con el sujeto agresor.

En esos términos, distinguió al sujeto agresor del sujeto agredido, y especificó el vínculo particular de hombres y mujeres para presentar este esquema: 1) Asesinato de hombre contra hombre; 2) Asesinato de hombre contra mujer: a) Feminicidio; b) Asesinato no feminicida; 3) Asesinato de mujer contra mujer; 4) Asesinato de mujer contra hombre(Russell, 2006a, p. 75). Es una noción incipiente que llevará a precisiones importantes, como veremos.

Al tenor de lo dicho, producto de más de una década de trabajo académico, científico y político por parte de mujeres mexicanas observando y analizando críticamente la violencia exacerbada en Ciudad Juárez, frontera de México con Estados Unidos, así como en el eje analítico-conceptual aportado por Diana Russell con la noción anglosajona Femicide, la acuñación del término Feminicidio, se convirtió en punto de inflexión político-conceptual y social y amplió la comprensión de la privación de la vida de mujeres por razones de género.

A decir de Marcela Lagarde (2006a, p. 20), traducir de manera directa el término femicidio, minimiza y reduce la violencia en contra de las mujeres a un acto ejercido al sexo de la persona: "es una voz homóloga a homicidio y sólo significa asesinato de mujeres" (p.20). Al emplear feminicidio en estos términos, se pone en el escenario la violencia política de género, así como la necesidad de reconocer que, derivada de los procesos de socialización anclados en la biología de los cuerpos, la interpretación social que se hace de la misma y que produce desigualdad e injusticia para las mujeres, hace partícipe al Estado y sus instituciones a reconocerse como sujetos obligados de atención a esta práctica, ya que no solamente se está en presencia de violencia institucional, sino ante la ruptura de los procesos de democratización de la vida social ${ }^{5}$.

En acuerdo con la misma autora, el feminicidio es el acto de matar a una mujer por su condición de género; la formulación de este concepto implica una dimensión política cuyo

\footnotetext{
${ }^{5}$ Marcela Lagarde ha reiterado (2006a; 2006b; 2008) la necesidad de confirmar el carácter político del feminicidio para evitar su lectura en clave sexual. Zillah Eisenstein (2008) emplea la noción señuelo sexual para advertir el peso de la dimensión sexual como factor explicativo de la violencia y ocultar condiciones estructurales de orden económico-político. Izabel Solyszko (2013) indica la irrelevancia del rasgo semántico y pone el acento en tres ejes conceptuales: genérico, singular y jurídico.
} 
propósito es denunciar la falta de respuesta y el incumplimiento estatal de sus obligaciones internacionales de garantías, entre ellas investigar y sancionar. Citada por Atencio y Laporta (2012, s/p), Lagarde considera que el feminicidio es un crimen de Estado, es decir, se trata de "una fractura del Estado de derecho que favorece la impunidad" y va en detrimento de la libertad y la seguridad de las mujeres. El concepto abarca el conjunto de hechos que caracterizan los crímenes y las desapariciones de niñas y mujeres en casos en que la respuesta de las autoridades sea la omisión, la inercia, el silencio o la inactividad para prevenir y erradicar estos delitos.

En acompañamiento al trabajo de Diana Russell y Marcela Lagarde, otras autoras con énfasis específicos enriquecen y profundizan el entendimiento del fenómeno feminicida. Izabel Solyszko (2013) identifica a Julia Monárrez Fragoso, Lucía Melgar y Rita Laura Segato como exponentes de tres líneas de construcción conceptual, a saber: genérica, singular y jurídica.

La concepción genéricaasocia la condición de género y misoginia que enmarca los actos de violencia exacerbada (letal) en contra de las mujeres desde un sistema patriarcal de organización del mundo. La concepción singular remite a la necesidad de identificar las circunstancias particulares que conducen a la muerte violenta de las mujeres, al tiempode interrogar respecto a situaciones como secuestros, asesinatos, y victimización posterior sobre los cuerpos de las mujeres, para transitar del feminicidio sexual serial a feminicidio sexual sistémico, íntimo, por ocupaciones estigmatizadas (estas aproximaciones consideran que la violencia feminicida no se explica solamente por la estructura patriarcal). La concepción jurídica sitúa la discusión más allá de la precisión técnica y judicial por la que se puede determinar la existencia del fenómeno, para pasar a un ejercicio penal efectivo que garantice la seguridad de las mujeres; la noción de femigenocidio permitiría identificar claramente que la violencia exacerbada que clausura la vida de las mujeres distingue entre la violencia puntual (en ámbitos domésticos) y la violencia estructural (en ámbitos impersonales).

\section{El Estado mexicano ante el feminicidio}

Se han conformado al interior de la estructura institucional del gobierno mexicano, instancias mandatadas para observar sistemáticamente el impacto de la violencia de género en las experiencias de vida de las mujeres con la finalidad de suprimirla. A ese propósito, la Comisión nacional para prevenir y erradicar la violencia de género en contra de las mujeres (CONAVIM), 
dependiente de la Secretaría de Gobernación, señala en su sitio oficial de Internet (https://www.gob.mx/conavim)que, en cumplimiento a las disposiciones contenidas en el Artículo 1 de la Ley general de acceso de las mujeres a una vida libre de violencia, promulgada el 1 de febrero de 2007, las autoridades de los diferentes niveles de gobierno (federal, estatales y municipales) están comprometidas a:

prevenir, sancionar y erradicar la violencia contra las mujeres, así como los principios y modalidades para garantizar su acceso a una vida libre de violencia que favorezca su desarrollo y bienestar conforme a los principios de igualdad y de no discriminación, así como para garantizar la democracia, el desarrollo integral y sustentable que fortalezca la soberanía y el régimen democrático establecidos en la Constitución Política de los Estados Unidos Mexicanos.(Congreso General de los Estados Unidos Mexicanos, 2007a)

En ese marco formal, el Código Penal Federal, en su Artículo 325, dice del feminicidio:

Comete el delito de feminicidio quien prive de la vida a una mujer por razones de género. Se considera que existen razones de género cuando concurra alguna de las siguientes circunstancias: a) La víctima presente signos de violencia sexual de cualquier tipo; b) A la víctima se le hayan infligido lesiones o mutilaciones infamantes o degradantes, previas o posteriores a la privación de la vida o actos de necrofilia; c) Existan antecedentes o datos de cualquier tipo de violencia en el ámbito familiar, laboral o escolar, del sujeto activo en contra de la víctima; d) Haya existido entre el activo y la víctima una relación sentimental, afectiva o de confianza; e) Existan datos que establezcan que hubo amenazas relacionadas con el hecho delictuoso, acoso o lesiones del sujeto activo en contra de la víctima; f) La víctima haya sido incomunicada, cualquiera que sea el tiempo previo a la privación de la vida; g) El cuerpo de la víctima sea expuesto o exhibido en un lugar público.

De las consideraciones jurídicas a este propósito, se desprende el dispositivo de acción Alerta de violencia de género contra las mujeres (AVGM) (Congreso General de los Estados Unidos Mexicanos. (2007b)), el cual supone la identificación de un conjunto de condiciones que atentan contra la integridad vital de las mujeres, así como el cumplimiento de un protocolo burocrático previo a su implementación, a saber: 
1) organismos de la sociedad civil o de derechos humanos, presentan una solicitud de alerta de género ante la autoridad de gobierno local, estatal o federal;

2) aceptada la solicitud, se conforma el Grupo de Trabajo que atiende la solicitud, con la participación del Instituto Nacional de las Mujeres (Inmujeres), la Comisión nacional para prevenir y erradicar la violencia de género en contra de las mujeres (CONAVIM), Comisión Nacional de Derechos Humanos (CNDH), más dos académicos nacionales y dos locales;

3) el Grupo de Trabajo realiza una visita de campo, a partir de la cual informa al ejecutivo estatal sobre las medidas a llevar a cabo en un plazo no mayor a seis meses;

4) concluido el término establecido y a partir de las acciones desarrolladas por la autoridad estatal, el Grupo de Trabajo emite un dictamen que dirige a la Secretaría de Gobernación para que ésta declare (o no) la Alerta de violencia de género contra las mujeres (AVGM);

5) en caso afirmativo, la Secretaría declara la Alerta de violencia de género contra las mujeres (AVGM), al tiempo de indicar las acciones preventivas por parte de las autoridades de gobierno estatal y municipales y su delimitación territorial.

El 7 de julio de 2017, mujeres del estado de Puebla vieron denegada su solicitud de alerta de género emplazada en 2016, misma que fue revocada por un juzgado federal el pasado 8 de abril de 2019; en la resolución judicial se indicaron " 45 medidas de prevención, seguridad y justicia", las cuales se resumen en la promoción del derecho "a la justicia pronta, expedita e imparcial; la prestación de servicios jurídicos, médicos, psicológicos especializados y gratuitos, además de la rehabilitación y la reparación del daño de las víctimas", mediante la difusión, a través de diferentes medios y con el involucramiento de diversos actores sociales y políticos, de un mensaje del gobierno del estado de "cero tolerancia" (CONAVIM). (2019).LaAlerta de violencia de género contra las mujeres-Puebla incluyó 50 municipios $^{6}$, casi una cuarta parte de

\footnotetext{
${ }^{6}$ Acajete, Acatlán, Acatzingo, Ajalpan, Amozoc, Atempan, Atlixco, Calpan, Chalchicomula de Sesma, Chiautla, Chietla, Chignahuapan, Coronango, Cuautlancingo, Cuetzalan del Progreso, Huauchinango, Huejotzingo, Hueytamalco, Izúcar de Matamoros, Juan C. Bonilla, Libres, Los Reyes de Juárez, Ocoyucan, Oriental, Palmar de Bravo, Puebla, San Andrés Cholula, San Gabriel Chilac, San Martín Texmelucan, San Pedro Cholula, San Salvador
} 
los 217 que integran la entidad estatal, que comprometen a las autoridades estatal y municipales a prevenir, sancionar y erradicar la violencia de género para octubre de 2019, mediante informe que, eventualmente aprobaría la Secretaría de Gobernación.

\section{Aproximación metodológica y referentesde contexto}

Los hallazgos aquí presentados forman parte de una primera etapa de investigación, cuyo propósito general se orienta a identificar singularidades en los vínculos entre economía, pobreza y violencia de género feminicida. Empleamos una perspectiva cualitativa de interpretación sobre la base de información periodística, a partir de la cual trazamos perfiles de victimarios y víctimas, situamos espacialmente los casos de feminicidio y describimos condiciones socioeconómicas generales que enmarcan tales espacios.

De manera puntual, atendemos la exposición de estos casos en medios periodísticos que, si bien no es su intención explícita, dejan ver la presencia de factores estructurales ligados con representaciones de género dominantes desde las que se consiente socialmente la violencia de género hacia las mujeres. Así, los insumos de este artículo se apoyan en la compilación de notas provenientes de seis diarios localesdigitales: Central (www.periodicocentral.mx), Diario Cambio (www.diariocambio.com.mx), e-consulta (www.e-consulta.com), El Popular (www.elpopular.mx), La Jornada de Oriente (www.lajornadadeoriente.com.mx), Puebla on line (https://pueblaonline.com.mx).

Se trata de medios independientes con orientaciones ideológicas y políticas diversas, los cuales enfatizan o minimizan aspectos de interés público y con ello, dificultan el seguimiento sistemático de las notas de interés: dado que no todos los medios reconocen como feminicidio los mismos casos, subsanamos este obstáculo a partir de crear una base de datos integrada por 36 variables, que al momento de esta presentación acumula 476 casos provenientes desde el año 2013, en la que establecimos vínculos diversos entre acto feminicida, víctima y victimario, los cuales se condensan de la siguiente manera:

EL Seco, Santiago Miahuatlán, Tecali de Herrera, Tecamachalco, Tehuacán, Tepanco de López, Tepatlaxco de Hidalgo, Tepeaca, Tepexi de Rodríguez, Tepeyahualco, Teziutlán, Tlacotepec de Benito Juárez, Tlaltenango, Tlaola, Tlapanalá, Tlatlauquitepec, Zacapoaxtla, Zacatlán, Zaragoza, Zoquitlán. 
a) Contexto. Incluye año, municipio, lugar en el que se encontró el cuerpo, fecha del posible fallecimiento, número de carpeta en investigación judicial (documentada en el mínimo de los casos), fecha de publicación y periódico digital en el que se registra el hecho;

b) Víctima.Destacanombre y edad (muchas veces la víctima se define en calidad de desconocida), estado civil, con ( $\sin$ ) hijas(os) (información presente cuando el victimario era pareja sentimental de la víctima); por último,

c) Victimario. Datos similares a los de la víctima, excepto por el hecho de que, a lo largo de la documentación revisada, se evidencia la carencia de datos al grado del encubrimiento de identidad (excepto cuando este mantiene algún vínculo afectivo con la víctima).

Al observar la síntesis del conjunto informativo, nos percatamos que los expedientes de los victimarios despliegan menor información comparada con la de las víctimas. Pensamos que tal situación puede explicarse por la justificación colectiva de la que no escapan autoridades y medios, y su traducción en lesiva certeza social: que las mujeres son responsables de desatar la violencia en contra de sí mismas.

Así, anclados en la representación viril de la violencia y de la sexualidad, mientras los medios hacen apología de la violencia de género, a partir de reunir y adicionar tantos aspectos como sean necesarios para dar un tono morboso a la nota, y presentan cifras no siempre concordantes con las presentadas al público por parte de la autoridad gubernamental, esta, a pesar de la existencia de tipificaciones penales vinculadas con los feminicidios, así como del aumento en el número de casos, no siempre los reconoce públicamente pues suponen un hándicap político en su contra y no está dispuesta a activar la alerta de género.

A partir de los criterios señalado arriba, presentamos 17 casos (ver Mapa 1. Localización geográfica de feminicidios en la ciudad de Puebla); los hemos situado en el espacio territorial en que se presentaron durante la temporalidad observada (enero 2018-abril 2019) y superpuesto con datos asociados a condiciones socioeconómicas de pobreza en la ciudad de Puebla (ver Mapa 2. Mapa interactivo del Consejo Nacional de Evaluación de la Política de Desarrollo Social). 
Al visualizar ambos mapas, hemos encontrado coincidencias con planteamientos de diferentes autoras, quienes han manifestado la presencia de estrechos vínculos entre la forma dominante de la economía y la cultura que organizan la vida social, caracterizada por procesos de feminización del trabajo y de la pobreza; señalando, también, que en ellos se expresa una creciente polarización asociada a la distribución global de riqueza, sostenida por mecanismos de traslape que dificultan ver procesos históricos de larga duración actuando de manera puntual sobre los cuerpos de las mujeres, al tiempo que se asientan en agresivas modalidades de economicismo, androcentrismo e individualismo (Fraser, 2020; Amorós, 2005). Asimismo, que en los denominados «nuevos-viejos» escenarios (Eisenstein, 2008; Eisenstein, 1997), la explotación global de clase hace acto de presencia contra aquellos sujetos quienes, con su presencia dinámica en estos escenarios, ponen en duda la democracia global (Benería, 2005; Ackerly, 2000).

Es con las aportaciones de este conjunto variado de autoras que al identificar en esta primera etapa la existencia de dos fenómenos visualmente traslapados, los hemos tomado como base del presente análisis, el cual apunta a condiciones de precariedad económica y ejercicio de la violencia en espacios periféricos de la zona de estudio.

\section{Puebla: espacio óptimo para prácticas feminicidas}

El Anuario estadístico y geográfico de Puebla 2017 (Instituto Nacional de Estadística y Geografía, 2017), muestra a la ciudad de Puebla ubicada en la zona centro de la república mexicana, a 107 kilómetros en línea recta al oriente-sur de la ciudad de México, capital del país: con 1'576.259 habitantes (829.525 mujeres y 746.734 hombres) para el año 2015, era considerada la quinta ciudad más poblada a nivel nacional. Su configuración como zona metropolitana acrecienta su demografía y complejiza su caracterización socioeconómica. La cercanía con la capital de la república, la convierte en polo de atracción para pequeñas poblaciones del estado y de otras entidades que, dedicadas a actividades productivas diversas, compiten entre sí por los escasos recursos y favorecen la lógica de explotación laboral y acumulación de riqueza. Entre las anteriores, destaca un conjunto de ramas de producción y de sujetos económicos asociadas a ella: industrias automotrices globales (Volkswagen y Audi), 
maquilatextil, metalmecánica, química, de plásticos, alimentaria, servicios inmobiliarios, comercio al por menor, así como educativas públicas y privadas (Secretaría de Economía, 2018).

El estado de Puebla, región político-administrativa en que se ubica la ciudad de Puebla, forma parte de aquellas entidades, junto con Veracruz, Michoacán, San Luis Potosí, Yucatán y Campeche, consideradas de alta marginación ${ }^{7}$; de acuerdo con el Centro de Estudios de las Finanzas Públicas (2018).En ellas, el mercado laboral se distribuye entre 39\% de mujeres y $38 \%$ de hombres, respecto del total nacional; con relación a la población económicamente activa $(\mathrm{PEA})^{8}$, destacamos las siguientes cifras, a nivel nacional / estatal:

Tabla 1. Caracterización sociodemográfica del estado de Puebla en 2018

\begin{tabular}{|l|r|r|}
\hline Rasgo & Nacional (\%) & Estatal (\%) \\
\hline Año de escolaridad (8 - 12 años) & 10,0 & 9,5 \\
\hline Edad promedio (37.5 - 42 años) & 39,0 & 39,5 \\
\hline Población ocupada & 43,26 & 40,03 \\
\hline Población no económicamente activa & 3,22 & 9,55 \\
\hline Ingreso de 1 a 3 salarios mínimos & 47,29 & 46,23 \\
\hline Escolaridad (primaria y secundaria) & 71,24 & 75,64 \\
\hline Nivel de instrucción medio superior y superior & 47,10 & 54,18 \\
\hline Sector terciario & 60,93 & 53,42 \\
\hline Sector secundario & 25,80 & 25,91 \\
\hline Sector primario & 12,70 & 20,46 \\
\hline Posición subordinados y remunerados & 68,88 & 59,71 \\
\hline Posición empleadores & 4,73 & 5,10 \\
\hline Posición por cuenta propia & 22,18 & 26,24 \\
\hline No remunerados & 4,19 & 8,93 \\
\hline Tasa de desocupación & 2,11 & 3,14 \\
\hline Mayor desocupación por grupos de edad (25 -64 años) & 62,77 & 63,23 \\
\hline Sin acceso a instituciones de salud & 62,22 & 79,15 \\
\hline
\end{tabular}

${ }^{7}$ El Consejo Nacional de Población define marginación como: “fenómeno multidimensional y estructural originado, en última instancia, por el modelo de producción económica expresado en la desigual distribución del progreso, en la estructura productiva y en la exclusión de diversos grupos sociales, tanto del proceso como de los beneficios del desarrollo" (CONAPO, 2011).

${ }^{8}$ La Población económicamente activa se refiere a las "personas de 15 años o más que... tuvieron o realizaron una actividad económica" (CEFP, 2018). 
Fuente: elaboración propia con información del Centro de Estudios de las Finanzas Públicas (2018).

La Tabla 1 compara, grosso modo, información del contexto nacional y estatal, el cual resume un escenario apropiado para la reproducción cotidiana de la vida en condiciones de adversidad, representado por años de escolaridad escasos, edad productiva con baja inserción entre la población ocupada, alto registro como subordinada y alta proporción de desocupación.

Información a nivel nacional da cuenta de algunas diferencias por sexo: subordinados remunerados formales $(38,2 \%$ mujeres y $36,5 \%$ hombres $)$, subordinados remunerados informales (30,4\% mujeres y $32,6 \%$ hombres) independientes informales remunerados $(20,4 \%$ mujeres y $21,1 \%$ hombres), independientes formales remunerados (4,8\% mujeres y $6,9 \%$ hombres) y no remunerados $(6,3 \%$ mujeres y $2,9 \%$ hombres $)$.

Este escenario se asienta en el proceso de configuración de una división internacional del trabajo capitalista, definida como capitalismo organizado de Estado y nuevo capitalismo desorganizadotransnacional (Fraser, 2008), constituida en lo material por la polarización económico-política a la que crecientes sectores de población, usualmente no calificados laboralmente, se ven forzados a su inserción en el sector servicios para satisfacer las necesidades de minorías poseedoras de capitales de distinto orden, al tiempo que favorece una ideología de corte neoliberal, alrededor de la cual el esfuerzo individual es capaz de impulsar y concretar logros materiales y subjetivos. Se trata del orden de vida basado en la violencia permanente que demanda ingentes energías por parte de los individuos para afrontar demandas que los trascienden (Morini, 2014; Sassen, 2014; Weeks, 2011; Gargallo, 2009).

Como señalamos arriba, los hallazgos presentados, corresponden a la fase iniciada en enero de 2018 y concluida en abril de 2019 con un recuento total de 119 feminicidios: 82 casos en 2018 con un promedio de 6,8 por mes y 37 casos de enero a abril del 2019 con un promedio de 9,25 por mes. El referente de edad fue de 24 a 26 años. En los casos que se especifica el estatus civil, la mayoría de las mujeres se encontraba casada, en menor proporción sostenían relaciones de unión libre y eran madres solteras. Se identificó esta condición porque el presunto responsable del asesinato fue el marido o pareja sentimental de la víctima. Finalmente, detectamos características relacionadas al lugar en el que se realizan los feminicidios o se encuentran los 
cuerpos de las víctimas; estos remiten a las periferias de la ciudad, así como municipios de la entidad que son referidos en la alerta de violencia de género.

A partir de lo anterior, elaboramos un mapa de distribución de casos en el período mencionado (ver Localización geográfica de feminicidios en la ciudad de Puebla).Al contrastarlo con el Mapa interactivo delConsejo Nacional de Evaluación de la Política de Desarrollo Social(CONEVAL) (Guadarrama, 2019), el cual da cuenta de los índices de pobreza extrema en Puebla, se reafirma la comprensión del feminicidio como una práctica conectada con condiciones económicas que facilitan formas de relación social de género signadas por la violencia.

Al superponer ambos mapas, es concurrente la intersección geográfica de casos de feminicidio con condiciones socioeconómicas ligadas a la carencia de servicios públicos, educación, vivienda y empleo. Dentro de los indicadores del Consejo Nacional de Evaluación de la Política de Desarrollo Social (CONEVAL), Puebla ocupa el segundo lugar con mayor pobreza a nivel nacional, tal como se refiere para las áreas periféricas de la ciudad, en las que se registran los casos de feminicidio reportados por los medios. 
Mapa 1. Localización geográfica de feminicidios en la ciudad de Puebla

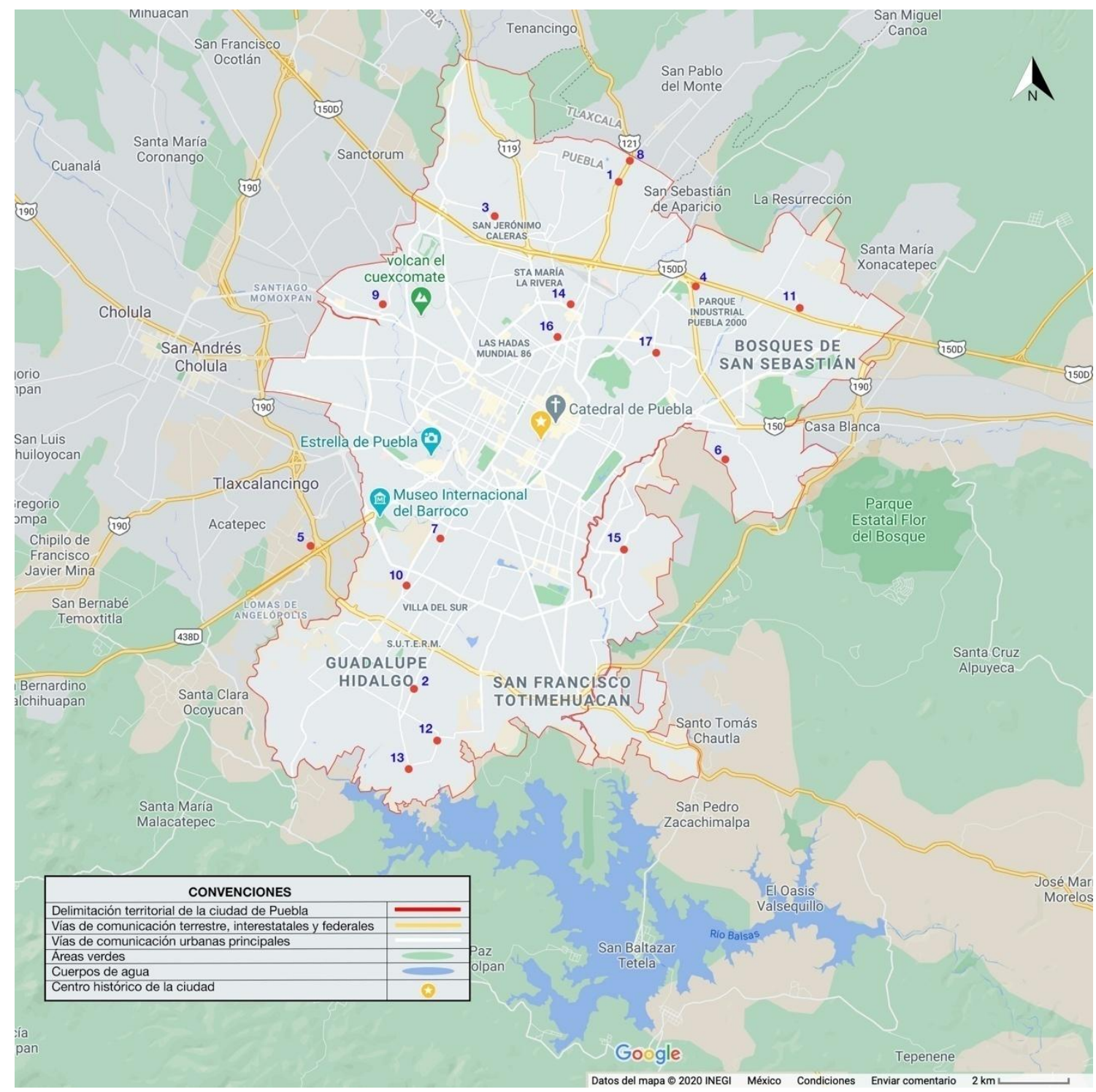

Fuente: Base de datos propia con información de los medios periodísticos indicados.

El mapa anterior registra los casos de feminicidio en el espacio territorial de la ciudad de Puebla; resalta la limítrofe municipal en color rojo, los casos con un punto en color rojo y su numeración en color azul (La aplicación Google Maps traza líneas de demarcación territorial donde no corresponden y ubica el caso 5 fuera de la traza de la ciudad). La información periodística permite situar el lugar en el que las mujeres fueron privadas de la vida o fueron encontradas muertas; en ocasiones se alude al carácter "popular" del espacio en que se produjo la privación de la vida: urbanización, centros nocturnos, unidad médica en que fue atendida la 
víctima; ocasionalmente, no se sabe en qué lugar fue privada de la vida. A continuación, enlistamos los casos en seguimiento:

1. María 9 . Infonavit El Conde. Febrero, 2018.

2. Mónica. Balcones del Sur: Bar Noa Noa. Enero, 2018.

3. Monserrat. Hospital de Ortopedia. Marzo, 2018.

4. Mariana. Gonzalo Bautista: domicilio particular. Mayo, 2018.

5. Marisa. San Isidro: vía pública. Mayo, 2018.

6. Michelle. Lomas de Chapultepec. Julio, 2018.

7. Marypaz. 13 a Sur: San José Mayorazgo. Septiembre, 2018.

8. Maricarmen. San José el Conde: domicilio particular. Septiembre, 2018.

9. Mariand. Boulevard Forjadores: Motel El Parador. Octubre, 2018.

10. Melisa. 11 sur y 105 poniente: obra negra. Octubre, 2018.

11. Melanie. Valle de las Flores: vía pública. Enero, 2019.

12. Mercedes y Micaela. Colonia Santa Lucía: vía pública. Marzo, 2019.

13. Mimí. Jardines del sur: domicilio particular. Marzo, 2019.

14. Mayela. Colonia 20 de noviembre: vía pública. Marzo, 2019.

15. Meg. Colonia La María: vía pública. Abril, 2019.

16. Mia. Colonia Santa María: domicilio particular. Abril, 2019.

17. Maya. Infonavit El Conde: domicilio particular. Abril, 2019.

\footnotetext{
${ }^{9}$ Para proteger el anonimato de las víctimas y evitar su re-victimización, se emplean nombres ficticios.
} 
Mapa 2. Mapa interactivo del Consejo Nacional de Evaluación de la Política de Desarrollo Social (CONEVAL)
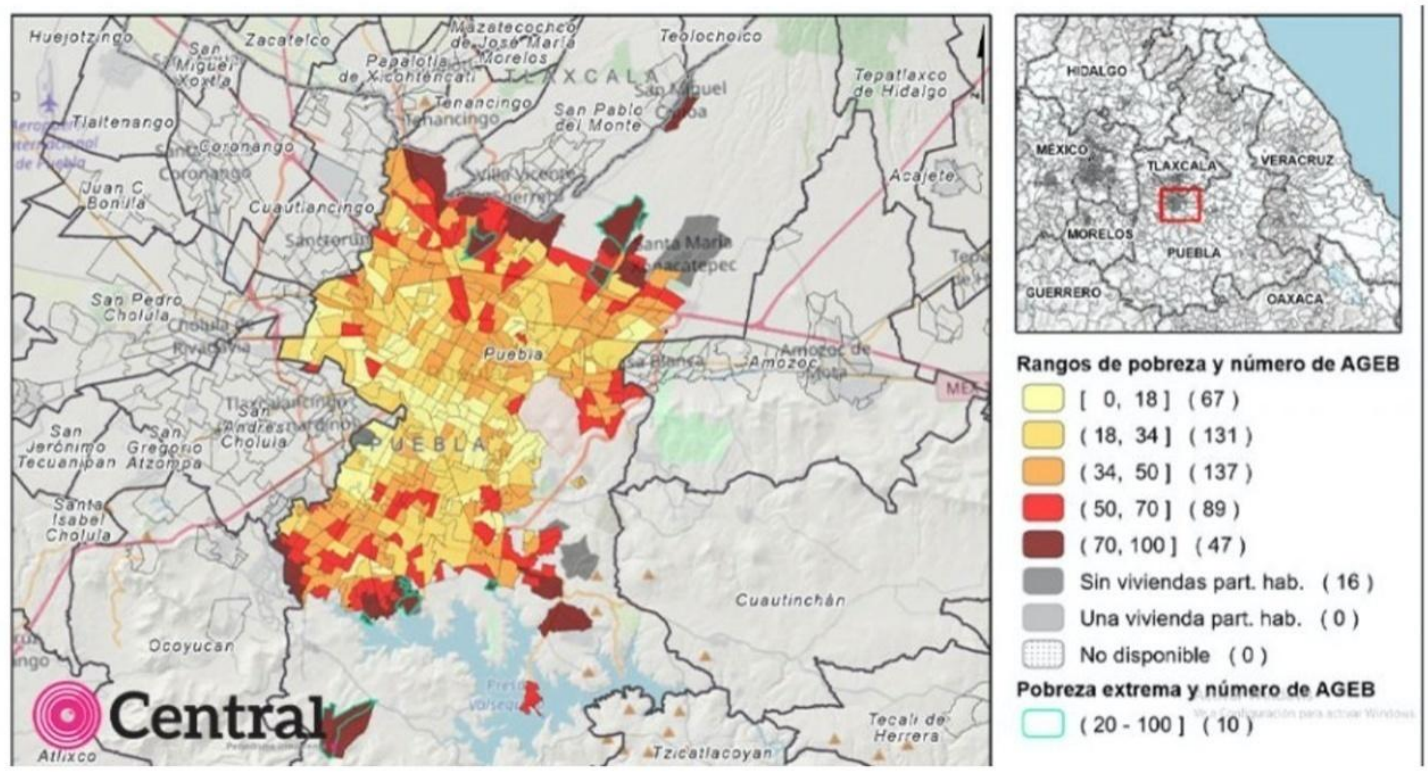

Fuente: Guadarrama (2019).

Nuestro supuesto de que la violencia de género se exacerba en contextos de precarización económica como consecuencia del modo capitalista y patriarcal de regulación de la vida social, ha sido considerado por autoras ya mencionadas. Celia Amorós (2005) nos recuerda que "la globalización neoliberal ha incrementado la feminización de la pobreza, ha generado el deterioro de la calidad de vida de las mujeres por los recortes y desmantelamientos del Estado del Bienestar, el incremento de los puestos de trabajo femeninos - aunque no sólo- en la economía sumergida e informal" (p. 25). Lourdes Benería (2005), por su parte, problematiza la relación trabajo-género al reconocer que contribuye a mejorar las condiciones generales de vida de las mujeres, aunque también las expone a un grado mayor de prácticas sexistas. Hester Eisenstein (2009) sitúa la centralidad del trabajo de las mujeres en el proceso de globalización económica como factor que las convierte en un nuevo proletariado por la vía de la eliminación del salario familiar, pero también por el desmantelamiento de la red de salud pública. En contraparte, Brooke Ackerly (2000) resalta el caso de mujeres rurales en procesos de desarrollo local, para quienes el factor económico es un elemento de tensión que no se expresa en violencia, siempre y cuando esté presente la experiencia de empoderamiento de género colectivo. 
Señalamos lo anterior bajo la consideración de una primera lectura al complejo fenómeno de la relación entre economía, pobreza y violencia de género; en la información visual presentada convergen espacialmente condiciones socioeconómicas asociadas a la pobreza con los casos de feminicidio identificados que nos llevan a pensar en, primera instancia, en esa relación. Habrá que profundizar en el análisis de la dimensión ideológica para reconocer el papel de la representación de las mujeres como sujetos económicos, de sus cuerpos como objetos de violencia, de modo de identificar la articulación de ambas dimensiones en la producción de violencia feminicida.

Reconocer la existencia de la violencia de género y de expresiones puntuales como el feminicidio, sintetiza un proceso histórico de reciente data en el contexto mexicano, el cual tiene sus orígenes feministas en el movimiento político internacional de mujeres del último tercio del siglo $\mathrm{XX}$, reivindicador de la configuración de vida social, económica y política mediada por la democracia de género. Sin esa demanda, las condiciones estructurales de desventaja que el genérico de las mujeres afronta de manera diaria, serían mayores yse mantendrían veladas. La lenta pero continua transformación de esta situaciónhace emerger expectativas que, eventualmente convertirán en hecho lo que hasta ahora aún forma parte de un proyecto civilizatorio en ciernes.

\section{Producción sociopolítica de espacio y género}

Ha sido constantela noción del espacio como punto de referencialocalizado en el mundo de la naturaleza, y, por ende, como receptáculo neutro, pasivo o paralelo a la experiencia vital humana. En franca oposición a esa idea, señalamos que no solamente está dotado de género, sino que el poder se inscribe en él y adscribe a los sujetos que lo habitan, es difusor de disposiciones reflexivas y prácticas entre las personas como consecuencia de un proceso de larga data no sensible a primera vista a nuestro entendimiento ${ }^{10}$.

\footnotetext{
${ }^{10}$ Sutiles implicaciones se derivan de categorías emparentadas como espacio, lugar, territorio, las cuales, según su singular formulación teórica, las alejan de o aproximan a la acción humanamente configurada; en este momento, es innecesario abundar en tales diferencias/semejanzas, en la medida en que nos interesa su carácter históricamente construido, y para la perspectiva feminista resultan relevantes en el análisis de las relaciones sociales de género: el topos y la distribución disimétrica de poder y sus efectos concomitantes en las experiencias vitales de mujeres y hombres.
} 
En acuerdo con Vianello y Caramazza (2002), la aportación principal de la geografía feminista a la comprensión del espacio es su "crítica radical de las categorías fundamentales de las Ciencias Políticas tradicionales, en primer lugar, de la categoría de «territorio», que se suele asumir como uno de los elementos (neutrales) básicos de la organización política” (p. 21) y esadjetivado como prístino, inalterado por la acción humana. Extensión de esa noción apolítica se refleja en la percepción generalizada sobre el territorio, desde la cual la experiencia espacial o territorial es compartida de manera semejante por mujeres y hombres y no implica formas de desigualdad relativas a su acceso y uso, de tal suerte que el género resulta irrelevante en la comprensión de la dinámica social que ocurre en aquél.

Sin embargo, nuestra inmersión diaria en los variados ámbitos de sociabilidad, nos lleva a inferir que existen diferencias reales por razón de sexo, género, generación, clase, etnia, entre otras, así como de los particulares entrecruzamientos entre ellas, que limitan, habilitan o problematizan la forma en que estamos en o transitamos por aquellos: de manera amplia, tales diferencias suelen traducirse en inequidad y alcanzar niveles letales para sujetos focalizados.

Aportaciones feministas variadas así lo documentan, y en ellas identificamos vetas de análisis cultural y de la economía política relativas a la relación del territorio con el cuerpo, la sexualidad, el sexismo, la subjetividad, la interacción, la producción y el trabajo; en estas, la experiencia geográfica de género de las mujeres refiere un proceso histórico-social inscrito en dicotomías orientadas a la perpetuación de la desigualdad: hombres-mujeres, público-privado, político-privado, normalidad-anormalidad, producción-reproducción; movilidad-inmovilidad; seguridad-miedo; superordinado-subordinado (Fraser, 2020, 2015, 2003, 1990; Federici, 2018; Eisenstein, 2009; Ortiz, 2007;Howson, 2005; McDowell, 2000; Del Valle, 1997). En suma, el espacio condensa prácticas y representaciones que se sostienen, se contestan o se reformulan de modo continuo, en las que predominan situaciones de adversidad para las mujeres como grupo genérico.

Acudimos nuevamente a Vianello y Caramazza (2002), paraestablecerque la noción espacio:

deriva de la raíz indoeuropea pet, que denota la idea de abrir, desplegar, extender. El significado de crecimiento y expansión es más explícito en el sánscrito sphay, tanto así 
que en griego petannumi [petánnymi] significa «abarcar expandiendo» y en latín spatiumsignifica «lo que está siendo ampliado». La raíz es la misma que la de speed, que, antes que «velocidad», en inglés antiguo significaba «éxito». La idea de movimiento asociado a una empresa se halla, por tanto, implícita en el concepto de espacio y esta connotación fue la que se impuso en la sociedad creada por la élite gobernante, la masculina, con el resultado de que el espacio ha sido concebido en términos estratégicos. (p. 35)

Somos sensibles al impacto social que lo estratégico manifiesta en la vida con el esclarecimiento que Amparo Moreno Sardà (2007) hace respecto al término economía; oikonomía, en su original sentido aristotélico, refería la esfera doméstica a cargo de las mujeres, sin embargo, en algún momento se transformó cualitativamente a partir de la acción viril y su despliegue por el territorio con la finalidad del ejercicio político - el control— sobre los distintos recursos allí disponibles, incluidos los humanos. Siguiendo con esta autora, nos percatamos que esa expansión viril desvirtuó el anclaje original de la economía para desplazarla y, con ello, contribuir a la producción del sujeto varón como expresión y experiencia metonímica de la relación de nuestra especie con el entorno, es decir, como episteme de lo humano, narrativa que se propaga hasta nuestros días con la certeza de que su concepción representa la síntesis sensible y universal del mundo. El espacio, bajo esta línea argumentativa, impone a mujeres y hombres en los hechos, maneras diferenciales y desiguales de habitarlo (y de suponer la disposición de capacidades para ello).

A lo señalado por Amparo Moreno, Esther Pineda (2017) añade que se trata de un fenómeno de producción sociohistórica consistente en lo siguiente: las sociedades de filiación occidental configuran ambientes propicios para la inserción diferencial en el mundo hacia la disimetría de hombres y mujeres. La ontología de occidente es apta para ello, ya que "para la preservación de un estado relacional disímil entre hombres y mujeres han sido implementados una multiplicidad de dispositivos como el aparato religioso, el relato oral y el conocimiento científico; los cuales, al ser emanados de dios, la historia y la ciencia respectivamente, se harían incuestionables y en consecuencia inmodificables" (p. 11). Podemos deducir de lo anterior que la puesta en marcha de las relaciones entre los géneros, adquiere connotaciones previamente 
cargadas de significados que escapan a nuestra conciencia y permiten su validez sin cuestionamiento.

Con lo dicho hasta ahora, la relación entre espacio y poder se vuelve más transparente desde el análisis crítico de género; este tipo de aproximación interpretativa es relevante en la conformación de la apercepción colectiva del espacio, porque permite dar cuentadeque en la valoración de esas dimensiones de vida, la neutralidad no está ausente, al tiempo que fortalece el reconocimiento de la posición e inserción históricamente desiguales de los sujetos en la construcción de relaciones sociales en el mundo contemporáneo según su sexo, como veremos más adelante, como resultado de un proceso considerado deriva del mundo natural.

\section{El cuerpo como sustrato material y subjetivo de poder}

A las consideraciones sobre el espacio se asocian el cuerpo, su materialidad y representación. La perspectiva teórico-corporal y su inexorable lazo con los itinerarios corporales (Esteban, 2013) apuntalan la tesis del carácter ontológico integrador del cuerpo-género con su determinación espacio-temporal; el trayecto conceptual para entender su pertinencia en estudios como el que aquí compartimos (del embodiment, pasando por corporización y encarnación, a la incorporación, o también cuerpo o corporal), constituye referencia fundamental, pues da cuenta de la transformación de las definiciones diversas orientadas a aspectos singulares situados en las dimensiones del poder y la sexualidad, motivo que aquí reiteramos de manera explícita, además de aquellas presentes en indagatorias interesadas por otros campo como la medicina, la publicidad, la moda.

Destacamos dos posibilidades de análisis corporal, apoyadas en la idea de producción sociohistórica de los cuerpos, así como de sus límites y alcances según la condición de género del sujeto por conocer; por una parte, el referido al impacto estructural sobre el individuo que lo ata sin posibilidad de transformación y, por el otro, el referido a la capacidad de construcción del sujeto en la acepción de agente de su propia vida, circunstancia que favorece la posibilidad de cambio, aun en el marco de la aplastante vida social orientada por la subordinación, la exclusión, la explotación, la discriminación. Para Mari Luz Esteban (2013) es sutil y trascendente esta segunda distinción, ya que: 
Considerar a las personas en primera instancia como agentes de su propia vida y no exclusivamente como víctimas no significa que se piense que sus itinerarios se conforman de una manera lineal, plana, en oposición sin más a una orientación victimizadora clásica. [...Implica, además, prestar atención...] a las resistencias que esa misma enculturación corporal comporta, a la contestación y las transformaciones que los individuos ponen en marcha, consciente o inconscientemente, frente a su cultura. Supone, por tanto, no ocultar sino mostrar y contextualizar las contradicciones y conflictos y, en definitiva, la complejidad de las diferentes experiencias. (p. 14)

Resulta sugestiva la propuesta de Mari Luz Esteban de reconocimiento de los individuos como agentes de su propia vida; sin embargo, dadas las características documentales de nuestra indagatoria, no es susceptible de abordarse el fenómeno desde esta perspectiva, aunque sí de conjugarse con la idea de producción socio-histórica del cuerpo y del espacio ${ }^{11}$; más aún, de enfatizar de manera explícita la transformación cualitativa que implica conceptuar los cuerpos de las mujeres víctimas de feminicidio, no como objetos que las acompañan en su experiencia vital, sino como ese aspecto del complejo proceso de producción sociopolítica de sus formas de subjetivación del mundo.

En ese sentido, esa condición social e histórica inscrita en el género e incardinada por todas las personas, derivada del proyecto patriarcal de socialización de los géneros (Haug, 1988), lleva a las mujeres en tanto grupo genérico a estar expuestas, sometidas, sujeta(das) a prácticas de poder puestas en marcha por cuerpos-sujetos sexuados en clave viril, con todo y su voluntad individual de impedir que la violencia exacerbada se cebe sobre ellas.

Atendemos al señalamiento de Mari Luz Esteban respecto a la noción de agentes de su propia vida para referirse al reto que supone romper, conceptualmente, con la identidad de víctimavictimario que suele acompañar estereotipadamente a mujeres y hombres en las relaciones sociales de género marcadas por la violencia, al tiempo de indicar que ese rompimiento implica aceptar que las identidades de género se encuentran en constante transformación y en continuo movimiento, y que las víctimas se sitúan en un enmarañado escenario acotado por el conflicto y

\footnotetext{
${ }^{11}$ La no factibilidad de poner en marcha a plenitud esta perspectiva, se debe a que la información proveniente de las notas periodísticas ha congelado a los sujetos que describe, condición opuesta a la que Mari Luz Esteban demanda: sujetos de carne y hueso con los que se intersubjetiva dinámicamente.
} 
la búsqueda, consciente o no, de formas de salida a sus prácticas vitales mediadas por la violencia de género. Dicho de otra manera, no resulta adecuado prejuzgar la condición víctima-victimario de mujeres y hombres.

Sin embargo, se hace obligado atender la formulación desde una óptica diferente. Rita Laura Segato (2016) muestra la necesidad de hacer visible la condición de víctima de las mujeres en tanto sujeto de género producido históricamente: no para albergar la idea de debilidad y vulnerabilidad femenina unipersonal, dar por sentada la incapacidad de muchas de ellas para modificar su situación concreta, o determinar secamente que el individuo masculino particular incorpora en sus prácticas la voluntad de hacer daño, sino para señalar que existe una estructura de sociabilidad interiorizada colectivamente que coloca en planos de valoración desiguales lo femenino y lo masculino, y conduce a formas de ejercicio de violencia predominantemente representadas en los binomios mujer-víctima, hombre-victimario, como el mandato de violación (Segato, 2003), las cuales suelen pasar, desde una interpretación de sentido común misógino, como expresión comportamental derivada de la emergencia del salvaje social ${ }^{12}$ (Das, 2008), o de alguna afección o patología personal, expresión esta última que, de manera tajante, rechazamos ${ }^{13}$.

La discusión que se asoma tiene tintes de mayor profundidad que trascienden la primera impresión. Como la propia Mari Luz Esteban lo plantea con relación a su propuesta epistémica de los itinerarios corporales, y a partir de la indicación de Sherry B. Ortner (2019) ligada con la idea de la antropología comprometida, el abordaje de problemáticas como la violencia de género y su concreción económico-política en los sujetos-cuerpos de las mujeres, implica la mención ontológica y epistemológica propia de los abordajes feministas y críticos de género, de un sesgo en el que el escenario de la justicia social emerge como premisa necesaria para la transformación de las relaciones entre mujeres y hombres, y no solamente como un asunto asociado al deseo de cambio por parte del individuo(Ackerly y True, 2010).

\footnotetext{
${ }^{12}$ A este respecto, en su trabajo sobre la articulación entre violencia, género y subjetividad, Veena Das muestra que la presencia indetenible de este sujeto marginal que aterroriza a mujeres sin motivos racionales, convive con la incapacidad o complicidad estatal ante formas de violencia que, paradójicamente, le permiten mantener el control político de las poblaciones.

${ }^{13}$ Terry Eagleton (2019) aborda la patología personal o colectiva desde una perspectiva psicoanalítica no feminista, al referir las dificultades de entendimiento para la sociedad inglesa de principios de este siglo, alrededor de la muerte de un niño de 3 años de edad a manos de otros dos de 10 años. Se pregunta si el acto maligno es producto congénito o de la socialización, está mediado por la (in)conciencia individual o el contexto en que se insertan los infantes, o es una mixtura de ambas que requeriría el perdón, el castigo o ambos. El caso muestra la complejidad de la experiencia de subjetivación del mundo desde los itinerarios corporales asociada a la experiencia de la violencia.
} 
Así, la producción de una estructura de representaciones y prácticas del mundo montada sobre la imagen de determinantes biológicos aplicables a la especie entera, cuya síntesis expresiva y metonímica es el acto individual, muestra el carácter complejo de los análisis sobre la relación entre cuerpo (entendido como sujeto personificado), espacio y violencia, así como la sutil y obligada aproximación a aquella: sin desconocer que, en última instancia, los individuos construyen sus vidas día tras día, tampoco ignoramos que el mundo no existe allí para nosotras(os) desde la nada, sino que están presentes mediaciones sociopolíticas que nos guían a través de las cuales podemos interpretar la realidad en un modo significativo históricamente determinado.

Tal como nos recuerda Mari Luz Esteban (2013, la configuración generalizada de los sujetos acompañados de cuerpos e identidades, resulta importante para remarcar lo siguiente: "la identidad de género es siempre una identidad corporal, [...] nos identificamos en relación al [sic] género dentro y a partir de una determinada corporeidad, desde una vivencia y una percepción determinada de nosotros/as mismos/as como seres carnales"(p. 15). Con lo dicho hasta ahora respecto a la relación espacio-cuerpo-género, resulta asible el escenario en el que se despliega la violencia de género en los años recientes y, de manera puntual, el feminicidioen Puebla, México. En seguida expondremos el modo de aproximación al fenómeno referidoy posteriormente nos referiremosal contexto que rodea el ascendente número de casos en la entidad del centro del país.

\section{Comentarios finales}

El amplio debate conceptual sobre la violencia de género recoge tres grandes posturas asociadas al lugar de mujeres y hombres en sus relaciones sociales a lo largo de la historia: aquella que explica la diferencia sexual derivada de la representación patriarcal del mundo; la que remite a las condiciones económicas provistas por el capitalismo; y la que busca integrar las dos primeras, para reconocer la convergencia de lo cultural con lo económico. Aquí enfatizamos el proceso económico sin desconocer la importancia de las representaciones estereotipadas de género en la prolongación de prácticas violentas entre los géneros, en cuyo sostenimiento social los medios de información contribuyen de modo importante para su vigencia.

Como se pudo observar, la posición precaria de las mujeres es violenta de origen, en tanto el patriarcado es un modo de producción-reproducción cultural de los géneros signado por la 
exclusión femenina, en articulación con el modelo económico y sus relaciones de trabajo, el cual las integra en circunstancias de inequidad y explotación. Resultado de ambos procesos, la carga de trabajo se amplifica, pues a su obligada tarea histórica de administrar los recursos materiales y afectivos de los grupos domésticos, también se les fuerza a ser responsables de la generación de riqueza en el mundo público, extendiendo la continuidad funcional a su identidad con las tradicionales tareas de cuidado de los otros, situación que las ubica en un escenario dramático y cada vez más letal para ellas.

El vigente modelo de globalización económica instiga a las mujeres de manera ineludible a insertarse en espacios laborales históricamente masculinos y masculinizados; por efecto de su inserción a condiciones definidas estructuralmente por la precaridad $^{14}$, mujeres y hombres no disponen de cauces estructurales que den solución a la tensión de género, motivada por el sistema de ordenamiento socioeconómico actual; la interacción entre los géneros es ambigua, pues al tiempo que permite a las mujeres acceso a condiciones materiales y simbólicas progresivamente más favorables, también las sitúa en riesgo pues las convierte en amenaza a la estabilidad del arquetipo viril.

La revisión de las notas periodísticas sobre el fenómeno del feminicidio en el estado de Puebla (en la región central de México) durante el último año y medio, identifica elementos inexcusables a un posterior análisis de carácter etnográfico, entre los que deberemos observar aquellos asociados a ejes de diferenciación social, tales como edad y estado civil, así como a los espacios, pues estos, en tanto eje discursivo, práctico y analítico del feminicidio, cristalizan expectativas sociales sobre los cuerpos de mujeres y hombres y dan cauce a expresiones de vida singulares, usualmente marcadas por la tensión, la ambigüedad, el conflicto y la violencia.

El feminicidio hace visible uno de los contenidos filosóficos y ontológicos del proyecto civilizatorio feminista, el de la democratización de la vida social y económica; esta exigencia resulta problemática para el modelo patriarcal y androcéntrico ligado con la economía política

\footnotetext{
${ }^{14}$ La distinción entre precariedad y precaridad, implica, en acuerdo con Judith Butler (2010), para la primera, la condición biológica vulnerable de la especie humana, propia de los mamíferos superiores, que consiste en el lento proceso de adquisición de autonomía individual, el cual condiciona la necesidad de cuidados nutricios maternos. La segunda noción, alude a consideraciones de orden político respecto a la decisión institucional-estatal respecto a qué grupos o sectores sociales merecen ser atendidos y en qué nivel de prioridad por la estructura administrativa. Esta última noción, es de vital importancia, pues allí se refleja la ausencia de políticas públicas en materia de género y de aspectos económicos en relación con el sistema dominante de producción-reproducción.
} 
vigente, pues contesta la lógica de fragmentación del capitalismo contemporáneo y reclama la transformación de las mujeres en sujetos autónomos; sin embargo, este la elude de manera exitosa apelando al peso de la dimensión cultural.

Finalmente, en tanto proyecto comprometido, el feminismo orientado a la economía política nos impulsa a construir un nuevo orden simbólico y nombrar de otra manera el mundo, anticapitalista por principio, en el que las mujeres dejen de ser referenciadas por el servicio a los demás y los hombres por el ejercicio de la violencia. Un modo diferente impulsado por el sentido consciente de interdependencia y responsabilidad colectiva, en el que los factores asociados a la producción y la reproducción toman como eje relaciones de género que no penalizan identidades y prácticas en búsqueda de emancipación humana.

\section{Referenciasbibliográficas}

Ackerly, Brooke. (2000). Political Theory and Feminist Social Criticism. Nueva York: Cambridge University Press.

Ackerly, Brooke. y True, Jacqui. (2010). Back to the Future: Feminist Theory, Activism, and Doing Feminist Research in an Age of Globalization. Women'sStudies International Forum, (33), 464-472.

Amorós, Celia. (2005). La gran diferencia y sus pequeñas consecuencias... para las luchas de las mujeres. Madrid: Ediciones Cátedra - Universitat de Valéncia.

Atencio, Graciela y Laporta, Elena. (2012). Tipos de feminicidio o las variantes extremas de violencia patriarcal. https://feminicidio.net/articulo/tipos-de-feminicidio-o-las-variantesde-violencia-extrema-patriarcal

Benería, Lourdes. (2005). II. Globalización y género. En: GemmaCairó y Maribel Mayordomo (Comp.) Por una economía sobre la vida. Aportaciones desde un enfoque feminista (pp. 35-62). Barcelona: Icaria Editorial, S.A., Colección Más Madera.

Butler, Judith. (2010). Marcos de guerra. Las vidas lloradas. Buenos Aires: Paidós Editorial, Colección Contextos Ideas. 
Centro de Estudios de las Finanzas Públicas (CEFP). (2018). Caracterización del Mercado Laboral en México: Puebla. https://www.cefp.gob.mx.

Cámara de Diputados del H. Congreso de la Unión. (2020). Código Penal Federal. http://www.diputados.gob.mx/LeyesBiblio/pdf/9_010720.pdf.

Comisión Nacional para Prevenir y Erradicar la Violencia contra las Mujeres (CONAVIM). (2019). Declaratoria de Alerta de Violencia de Género contra las Mujeres para 50 Municipios del Estado de Puebla. https://www.gob.mx/conavim/prensa/declaratoria-dealerta-de-violencia-de-genero-contra-las-mujeres-para-50-municipios-del-estado-depuebla-196911?idiom=es

Congreso General de los Estados Unidos Mexicanos. (2007a). Ley general de acceso de las mujeres a una vida libre de violencia. Diario Oficial de la Federación, 1 de febrero de 2007. https://www.gob.mx/conavim/documentos/ley-general-de-acceso-de-las-mujeres-auna-vida-libre-de-violencia-pdf

Congreso General de los Estados Unidos Mexicanos. (2007b). Alerta de violencia de género contra las mujeres (AVGM).https://www.gob.mx/inmujeres/acciones-y-programas/alertade-violencia-de-genero-contra-las-mujeres-80739.

Consejo Nacional de Población (CONAPO). (2011). Capítulo 1. Concepto y dimensiones de la marginación.

http://www.conapo.gob.mx/work/models/CONAPO/Resource/1755/1/images/01Capitulo. pdf

Das, Veena. (2008). Violence, Gender, and Subjectivity. Annual Review of Anthropology, 37, 283-299.

DeKeseredy, Walter. (2011). Feminist Contributions to Understanding Woman Abuse: Myths, Controversies, and Realities. Aggression and ViolentBehavior, (16), 297-302.

Del Valle, Teresa. (1997). Andamios para una nueva ciudad. Lecturas desde la antropología. Madrid: Ediciones Cátedra, Universitat de València, Instituto de la Mujer. 
De la Fuente, María. (2015). Ideas de poder en la teoría feminista. Revista Española de Ciencia Política, (39), 173-193.

Eagleton, Terry. (2019). Sobre el mal. España: Ariel.

Eisenstein, Hester. (2009). Feminismseduced. How Global Elites Use Women's Labor and Ideas to Exploit the World. Boulder - Londres: ParadigmPublishers.

Eisenstein, Zillah. (1997). Lo público de las mujeres y la búsqueda de nuevas democracias. Debate Feminista, 15(8), 198-243.

Eisenstein, Zillah. (2008). Señuelos sexuales. Género, raza y guerra en la democracia imperial. Barcelona: Edicions Bellaterra.

Esteban, Mary. (2013). Antropología del cuerpo. Género, itinerarios corporales, identidad y cambio. Barcelona: Edicionsbellaterra, Serie General Universitaria 42.

Falquet, Jules. (2014). Hacia un análisis feminista y dialéctico de la globalización neoliberal: el peso del complejo militaro-industrial sobre las «mujeres globales». Revista Internacional de Pensamiento Político, (9), 139-148.

Federici, Silvia. (2018). El patriarcado del salario. Críticas feministas al marxismo. España: Traficantes de sueños, Colección Mapas 49.

Fraser, Nancy. (1990). ¿Qué tiene de crítica la teoría crítica? Habermas y la cuestión del género. En: SeylaBenhabib y Drucilla Cornell(Eds.). Teoría feminista y teoría crítica. Ensayos sobre la política de género en las sociedades del capitalismo tardío (pp. 49-88). España: Edicions Alfons El Magnànim, Institució Valenciana D’Estudis I Investigació, Colección Política y Sociedad 3.

Fraser, Nancy. (2003). ¿De la disciplina hacia la flexibilización? Releyendo a Foucault bajo la sombra de la globalización. Revista Mexicana de Ciencias Políticas y Sociales, XLVI (187), 15-33. 
Fraser, Nancy. (2008). El feminismo, el capitalismo y la astucia de la historia. New LeftReview, (56), 87-104.

Fraser, Nancy. (2015). Las contradicciones del capital y los cuidados. New LeftReview, (100), 111-132.

Fraser, Nancy. (2020). Los talleres ocultos del capital. Un mapa para la izquierda. Madrid: Traficantes de sueños, Colección Prácticas constituyentes.

Gargallo, Francesca. (2009). Feminismo y globalización: una mirada desde América Latina. En: Mariana Berlanga, Héctor Castañeda, y Armando Villatoro (Eds.). Mujer y violencia: el feminismo en la era de la globalización (pp. 87-102). México: Universidad Autónoma de la Ciudad de México, Cuadernos del Seminario 2.

Guadarrama, Nallely. (2019). Esta es la mancha del Coneval que pinta de rojo las zonas de pobreza extrema en la capital de Puebla. Central.Periodismo irreverente. https://www.periodicocentral.mx/2019/municipio/item/14760-esta-es-la-mancha-delconeval-que-pinta-de-rojo-las-zonas-de-pobreza-extrema-en-la-capital-de-puebla

Haug, Frigga. (1988). GenderRelations. En ShahrzadMojab (Ed.). Marxism and Feminism (pp. 33-75). Londres: Zed Books.

Howson, Alexandra. (2005). Embodying gender. Reino Unido: SAGE Publications.

Instituto Nacional de Estadística y Geografía (INEGI). (2017). Anuario estadístico y geográfico de Puebla. https://www.datatur.sectur.gob.mx/ITxEF_Docs/PUE_ANUARIO_PDF.pdf

Lagarde, Marcela. (2005). Los cautiverios de las mujeres: madresposas, monjas, putas, presas y locas. México: Universidad Nacional Autónoma de México, Colección Posgrado 8.

Lagarde, Marcela. (2006a). Introducción. Por la vida y la libertad de las mujeres. Fin al feminicidio. En: Diana Russell y RobertaHarmes (Ed.). Feminicidio: una perspectiva global (pp. 15-42). México: Centro de Investigaciones Interdisciplinarias en Ciencias y 
Humanidades-Universidad Nacional Autónoma de México, Colección Diversidad Feminista.

Lagarde, Marcela. (2006b). Del femicidio al feminicidio. Desde el jardín de Freud, (6), 216-225.

Lagarde, Marcela. (2008). Antropología, feminismo y política: violencia feminicida y derechos humanos de las mujeres. En: Margaret Bullen y Carmen Diez (Coords.). Retos teóricos y nuevas prácticas (pp. 209-239). España: Ankulegi.

McDowell, Linda. (2000). Género, identidad y lugar. Un estudio de las geografías feministas. Madrid: Ediciones Cátedra.

Moreno, Amparo. (2007). De qué hablamos y no hablamos cuando hablamos del hombre. Treinta años de crítica y alternativas al pensamiento androcéntrico. Barcelona: Icaria editorial, S.A., Colección Sociedad y Opinión.

Morini, Cristina. (2014). Por amor o a la fuerza. Feminización del trabajo y biopolítica del cuerpo. Madrid: Traficantes de sueños, Colección Mapas 39.

Ortiz, Anna. (2007). Hacia una ciudad no sexista. Algunas reflexiones a partir de la geografía humana feminista para la planeación del espacio urbano. Territorios, (16-17), 11-28.

Ortner, Sherry. (2019). Practicing Engaged Anthropology. Anthropology of This Century, (25) 119. http://aotcpress.com/articles/practicing-engaged-anthropology/

Pérez, Amaia. (2014). Subversión feminista de la economía. Aportes para un debate sobre el conflicto capital-vida. Madrid: Traficantes de sueños, Colección Mapas 40.

Picchio, Antonella. (2005). I. La economía política y la investigación sobre las condiciones de vida. En: GemmaCairó y Maribel Mayordomo (Comp.). Por una economía sobre la vida. Aportaciones desde un enfoque feminista (pp. 17-34). Barcelona: Icaria Editorial, S.A., Colección Más Madera. 
Pineda, Esther. (2017). Machismo y vindicación. La mujer en el pensamiento filosófico. Ciudad Autónoma de Buenos Aires: Prometeo Libros.

Radford, Jill. (1992). Introduction. En Russell, Diana. (Ed.),Femicide. The Politics of Women Killing (pp. 3-12). Nueva York: Twayne Publishers.

Rose, Gillian. (1993). Feminism and Geography. The Limits of Geographical Knowledge. ReinoUnido: PolityPress.

Russell, Diana. (2006a). Definición de feminicidio y conceptos relacionados. En Diana Russell y RobertaHarmes (Eds.). Feminicidio: una perspectiva global (pp. 73-96). México: Centro de Investigaciones Interdisciplinarias en Ciencias y Humanidades-Universidad Nacional Autónoma de México, Colección Diversidad Feminista.

Russell, Diana. (2006b). Conclusión. Feminicidio: la «solución final» de algunos hombres para las mujeres. En: Diana Russell y RobertaHarmes(Eds.). Feminicidio: una perspectiva global (pp. 345-366). México: Centro de Investigaciones Interdisciplinarias en Ciencias y Humanidades-Universidad Nacional Autónoma de México, Colección Diversidad Feminista.

Sassen, Saskia. (2014). Saskia Sassen on "before method" [Audio podcast]. http://methods.sagepub.com/podcast/saskia-sassen-on-before-method.

Secretaría de Economía (SE). (2018). Información económica y estatal: Puebla. https://www.economia.gob.mx.

Segato, Rita. (2003). Las estructuras elementales de la violencia. Ensayos sobre género entre la antropología, el psicoanálisis y los derechos humanos. Buenos Aires: Universidad Nacional de Quilmes.

Segato, Rita. (2016). La guerra contra las mujeres. Madrid: Traficantes de sueños, Mapas 45. 
Solyszko, Izabel. (2013). Femicidio y feminicidio: avances para nombrar la expresión letal de la violencia de género contra las mujeres. GénEros, Revista de investigación y divulgación sobre los estudios de género, (13), 23-41.

Vianello, Mino y Caramazza, Elena. (2002). Género, espacio y poder. Para una crítica de las Ciencias Políticas. Madrid: Ediciones Cátedra, Universitat de València, Instituto de la Mujer, Colección Feminismos 66.

Weeks, Kathi. (2011). The Problem with Work. Feminism, Marxism, AntiworkPolitics, and PostworkImaginaries. Estados Unidos: Duke UniversityPress. 